\title{
Correlation between transport properties and atomic configuration of atomic contacts of zinc by low-temperature measurements
}

\author{
E. Scheer, P. Konrad, and C. Bacca \\ Fachbereich Physik, Universität Konstanz, D-78457 Konstanz, Germany \\ A. Mayer-Gindner \\ Physikalisches Institut, Universität Karlsruhe, D-76128 Karlsruhe, Germany \\ H. v. Löhneysen \\ Physikalisches Institut, Universität Karlsruhe, D-76128 Karlsruhe, Germany \\ and Forschungszentrum Karlsruhe, Institut für Festkörperphysik, D-76021 Karlsruhe, Germany \\ M. Häfner \\ Institut für Theoretische Festkörperphysik, Universität Karlsruhe, D-76128 Karlsruhe, Germany \\ and Forschungszentrum Karlsruhe, Institut für Nanotechnologie, D-76021 Karlsruhe, Germany \\ J. C. Cuevas \\ Institut für Theoretische Festkörperphysik, Universität Karlsruhe, D-76128 Karlsruhe, Germany; \\ Forschungszentrum Karlsruhe, Institut für Nanotechnologie, D-76021 Karlsruhe, Germany; \\ and Departamento de Física Teórica de la Materia Condensada C-V, Universidad Autónoma de Madrid, E-28049 Madrid, Spain
}

(Received 24 August 2006; revised manuscript received 6 October 2006; published 27 November 2006)

\begin{abstract}
We report low-temperature measurements of the electrical transport properties of atomic contacts of the superconducting metal zinc, arranged with lithographically fabricated mechanically controllable breakjunctions (MCB) as well as with MCBs made from whiskers. The conductance histogram shows several peaks-not regularly observed for multivalent metals. The first peak - corresponding to the single-atom contact-is located slightly below one conductance quantum and is split into two subpeaks, indicating two preferred configurations of the single-atom contact. The existence of two configurations can also be traced by analyzing individual conductance vs distance curves of the MCB. The current-voltage characteristics in the superconducting state show nonlinearities due to multiple Andreev reflections (MAR), which we use for determining the transport channels. With the help of a tight-binding model for the transport channels we establish a correlation between the preferred conductance values and preferred atomic configurations.
\end{abstract}

PACS number(s): 74.50.+r, 73.40.Jn, 73.63.Rt

\section{INTRODUCTION}

For revealing and understanding the electronic transport properties of atomic scale circuits, atomic contacts are used as model systems. ${ }^{1}$ In this limit the electronic conduction can be attributed to a small number of independent electronic modes, nick-named "conduction channels." properties are fully described by a set $\left\{\tau_{i}\right\}=\left\{\tau_{1}, \tau_{2}, \ldots, \tau_{N}\right\}$ of transmission coefficients which depends both on the chemical properties of the atoms forming the contact and on their geometrical arrangement. The conductance of such a contact is given by the Landauer formula: $G=G_{0} \sum_{i=1}^{N} \tau_{i}$, where $G_{0}=2 e^{2} / h$ is the conductance quantum.

Experiments on a large ensemble of metallic contacts have demonstrated the statistical tendency of atomic-size contacts to adopt element-specific preferred values of conductance. The actual preferred values depend not only on the metal under investigation but also on the experimental conditions. However, for many metals, and in particular simple ones (like $\mathrm{Na}, \mathrm{Au}$, etc.) which represent in the bulk good free-electron systems, the smallest contacts have a conductance $G$ close to $G_{0}$ (Ref. 1). Statistical examinations of Al point contacts at low temperatures yield preferred values of conductance at $G=0.8 G_{0}, 1.9 G_{0}, 3.2 G_{0}$, and $4.5 G_{0}$ (Ref. 3), indicating that single-atom contacts of $\mathrm{Al}$ have a typical conductance slightly below the conductance quantum. However, it has been shown by the analysis of current-voltage (IV) characteristics in the superconducting state that $\mathrm{Al}$ single-atom contacts accommodate in general three transport channels, the transmissions of which add up to a value $0.8 \lesssim \tau \lesssim 1$ (Ref. 4). These findings are in agreement with measurements of the shot noise, ${ }^{5,6}$ conductance fluctuations, ${ }^{7}$ thermopower, ${ }^{8}$ and the supercurrent ${ }^{9}$ in such contacts. In particular, in the experiments on $\mathrm{Al}$, for a given contact geometry the transmission coefficients of the individual channels almost continuously change upon further deformation of the contact and no preferred transmission values have been found. ${ }^{4}$ A quantum-chemical model, ${ }^{10}$ which links the $\tau_{i}$ to the chemical valence and the atomic arrangement of the region around the central atom of the constriction, gives a prediction about the number of transport channels, but the $\left\{\tau_{i}\right\}$ depend on the precise atomic arrangement of the contacts. So far $\mathrm{Al}$ appears to be the only metal with the property to show peaks in the histogram close to multiples of $G_{0}$ although this does not correspond to quantized transmission values. However, since from the experiments no information about the 
atomic arrangement of the contacts can be deduced, a direct correlation between transport channels and configuration is still lacking. Therefore it is worthwhile studying a class of elements for which a similar transport behavior as found in Al could be expected but which offer simultaneously the possibility to correlate the transport properties to the crystal structure. So far, only for the monovalent metal Au signatures of different growing directions of nanowires could be found in the conductance traces by the combination of those measurements with transmission electron microscopy. ${ }^{11,12}$ However, in those experiments, no direct information about the conduction channels was available.

According to simplistic arguments divalent elements such as the alkaline-earth elements or the IIB subgroup elements $\mathrm{Cd}, \mathrm{Zn}$, and $\mathrm{Hg}$ should be insulating, since they have a completely filled outer $s$ shell. This apparent discrepancy to the experimental findings indicates that additional orbitals besides the $s$ orbitals contribute to the electronic conductance. Detailed calculations reveal that in the case of $\mathrm{Zn}$ the $4 p$ orbitals are the most relevant ones for a correct description of the bulk band structure. ${ }^{13}$ With these considerations, singleatom contacts of $\mathrm{Zn}$ are expected to have very similar transport properties as Al single-atom contacts. The distribution of the transmission coefficients, however, may deviate, since it depends on the exact atomic configuration. ${ }^{14}$ Since $\mathrm{Zn}$ crystallizes in a hexagonal, slightly distorted hcp structure while $\mathrm{Al}$ is a fcc crystal, the actual atomic arrangement of the point contacts might be different. Moreover, due to the "loss" of many neighbors, a single $\mathrm{Zn}$ atom in a contact may behave more atomiclike.

We will show that the conductance histogram of $\mathrm{Zn}$ shows several peaks, a fact that is unusual for multivalent metals. The first peak, which corresponds to the single-atom contact, is located slightly below one conductance quantum and is split-up into two subpeaks, which evidences the existence of two or more preferred configurations of the single-atom contact. We will demonstrate that indications for preferred atomic arrangements can be found in individual opening and closing traces of the junctions both measured in the normal as well as in the superconducting state. With the help of a tight-binding model for the transport channels we establish a correlation between the preferred conductance values deduced from the histograms and preferred channel transmissions which we use for linking the peaks in the histogram to preferred atomic configurations.

The paper is organized as follows: In Sec. II we describe the fabrication of lithographic MCBs and the measurement setup. Section III presents the experimental results obtained on these devices. In Sec. IV we introduce results from another type of breakjunction arranged with whiskers. In Sec. V we combine the results of both types of experiments with tight-binding calculations for the transport channels and propose a correlation between atomic structure and transport properties. Finally, Sec. VI summarizes the main conclusions.

\section{EXPERIMENT}

\section{A. Fabrication of lithographic $\mathrm{Zn}$ breakjunctions}

Here, we report at first the fabrication and lowtemperature electronic transport properties of lithographic

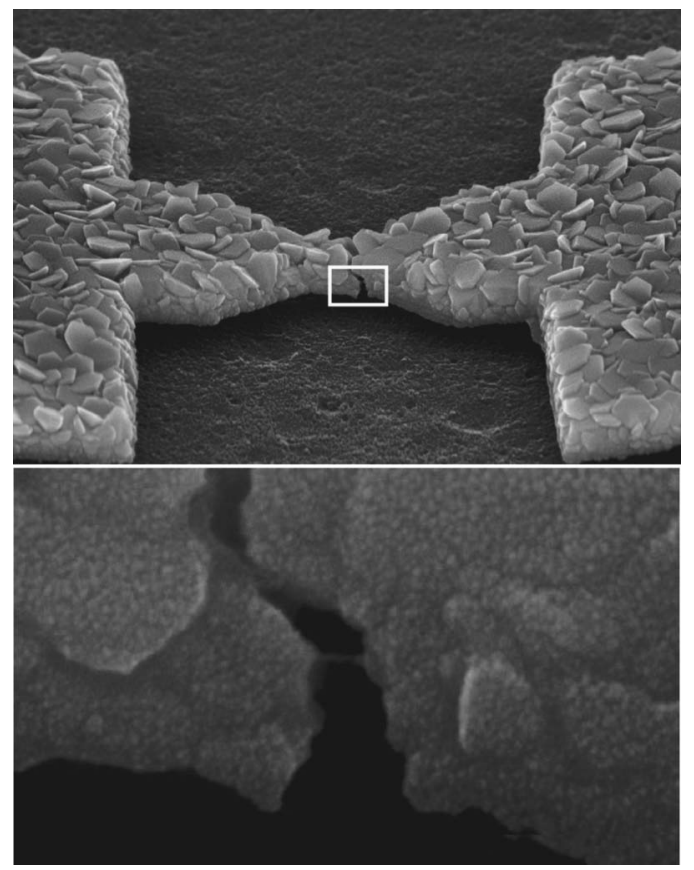

FIG. 1. Upper panel: Electron micrograph of sample No. 1 under an inclination angle of $70^{\circ}$, taken after measurement. The polycrystalline structure of the $\mathrm{Zn}$ film is clearly visible. The distance between the two wide electrodes is $2 \mu \mathrm{m}$. Lower panel: Magnification of the central part of the sample. A remaining spacing of the bridge arms of several $\mathrm{nm}$ and a fine nanowire between them is observed.

MCBs of $\mathrm{Zn}$ which we use for measuring the conductance histogram. The samples are fabricated using electron beam lithography along the lines of Ref. 15 . Our samples are $2 \mu \mathrm{m}$ long, $200 \mathrm{~nm}$ thick suspended $\mathrm{Zn}$ nanobridges, with a $200 \mathrm{~nm} \times 100 \mathrm{~nm}$ constriction in the center (cf. Fig. 1). The metal structure is deposited onto a polyimide layer that serves for planarization, electrical isolation from the metallic substrate and as sacrificial layer that is partially etched away for suspending the nanobridge. The thickness of the bronze substrates is $\approx 0.3 \mathrm{~mm}$, the distance between the countersupports of the bending mechanism is $10 \mathrm{~mm}$. With these sample parameters and the geometry of the mechanism we achieve reduction ratios (between the motion of the pushing rod and the displacement of the bridge arms) of the order of 5000:1 (see below). If $\mathrm{Zn}$ is evaporated at room temperature, it grows in islands of micron size with the hexagonal axis preferably perpendicular to the substrate plane. Since this structure is not suitable for the formation of atomic-size contacts, we take special care to reduce the grain size. We first deposit a $1 \mathrm{~nm}$ thick seed layer of Ge onto the substrate which is cooled by liquid nitrogen to a temperature of $\approx-50{ }^{\circ} \mathrm{C}$. At even lower substrate temperatures the different thermal expansions of the metallic substrate, the $\mathrm{Zn}$ itself, the polyimide sacrificial layer, and the polymer lithographic mask provoke cracks in either the mask or the $\mathrm{Zn}$ layer on warming up to room temperature. The metal is then evaporated from a home-made boron-nitride crucible heated by a tungsten foil at a pressure of $\approx 10^{-6}$ mbar and a rate of $\approx 0.2 \mathrm{~nm} \mathrm{~s}$ again at $\approx-50{ }^{\circ} \mathrm{C}$. The parameters are chosen 
empirically such that the grain size of the $\mathrm{Zn}$ layer does not exceed $\approx 200 \mathrm{~nm}$ and the adhesion is good enough for the subsequent lift-off process. Finally, the bridge is underetched in an isotropic oxygen plasma which reduces the height of the polyimide layer by about 300 to $500 \mathrm{~nm}$. At the narrow constriction the nanobridge is now unsupported. The resistance of the nanobridge does not increase during the etching process, indicating that reaction between the oxygen ions and $\mathrm{Zn}$ is weak.

\section{B. Transport measurements}

The bridge is then mounted on a three-point bending mechanism on a stick which is immersed into liquid helium and can be pumped down to reach temperatures of $1.5 \mathrm{~K}$. The residual resistivity ratio of the bridges is typically $\mathrm{RRR}=R(300 \mathrm{~K}) / R(1.5 \mathrm{~K}) \simeq 2.4$ from which we deduce an elastic mean free path of $13 \mathrm{~nm}$.

A screw with $500 \mu \mathrm{m}$ pitch, driven by a dc-motor on top of the cryostat through an (exchangeable) reduction gear box, controls the motion of the pushing rod that bends the substrate. The relative displacement of the pushing rod can be controlled to a precision of $\approx 4 \mu \mathrm{m}$, which due to the geometry of the bending mechanism results in a relative motion of the two anchor points of the bridge of around $0.9 \mathrm{pm}$. This was verified using the exponential dependence of the conductance on the interelectrode distance in the tunnel regime. The resistance of the sample is recorded by a fourpoint resistance bridge with a measuring current of $1 \mathrm{nA}$ and a resolution of $10^{-3}$ in a range from $200 \Omega$ to $200 \mathrm{k} \Omega$. Due to the mentioned texture of the evaporated film there are only few sliding planes parallel to the substrate which would allow for a stretching of the film. Consequently, it usually happens that the two electrodes remain separated by a gap of several nanometers after the first breaking. If this happens, in general no conducting contact can be established anymore. Only in 5 out of 20 samples was it possible to repeatedly close and open the bridge again. In these cases we often observe the formation of a nanowire in the electron micrograph (see lower panel of Fig. 1). Only when the first opening is performed very slowly $v<5 \mathrm{pm} / \mathrm{s}$ and when simultaneously measuring the conductance, the contact can be closed again. We assume that the measurement current in addition with the slow deformation supplies sufficient energy and gives enough time for the motion of the atoms to form the nanowire. Typical opening speeds for the later systematic measurements are $150 \mathrm{~nm} / \mathrm{s}$ for the pushing rod, corresponding to a displacement of the bridge-anchor points of $35 \mathrm{pm} / \mathrm{s}$, much slower than typical opening speeds of nanocontacts fabricated with the help of a STM or the "notchedwire" breakjunction technique. ${ }^{1}$ This limitation in opening speed also limits the number of opening traces that can be recorded within a reasonable time. The output of the resistance bridge is recorded by an oscilloscope and then transferred to a computer for calculating the histogram.

\section{RESULTS OBTAINED ON LITHOGRAPHIC BREAKJUNCTIONS}

\section{A. Conductance histograms}

When elongating the bridge, its conductance $G$ decreases in steps of the order of $1 G_{0}$, their exact sequence changing

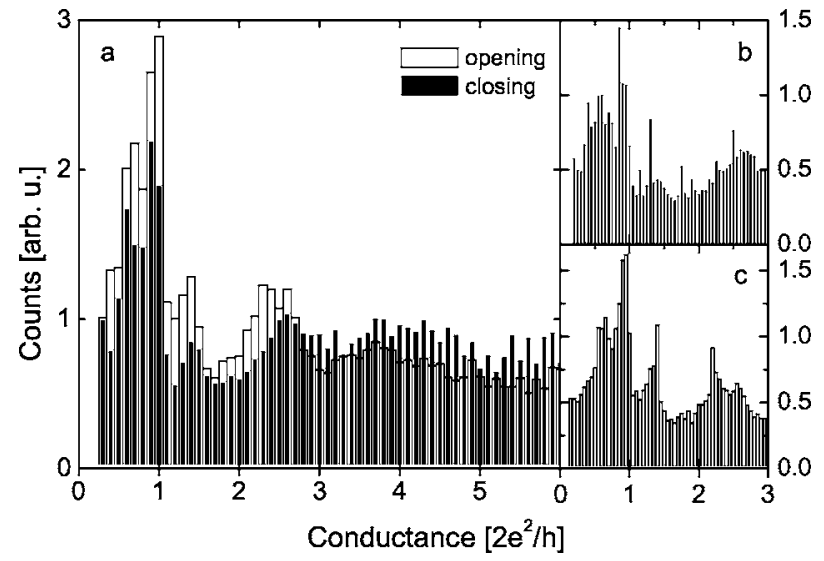

FIG. 2. (a), (b), and (c): Conductance histograms for opening and closing the contacts, recorded on sample No. 1 (2) at $T=1.5 \mathrm{~K}$. The histograms have been calculated from $\approx 600$ individual opening and closing curves, respectively.

from opening to opening (see Fig. 3). Since the mechanical deformation is different for opening and closing, we construct separate histograms for both experiments. Figure 2(a) displays the conductance histograms calculated from $\approx 600$ subsequent opening and closing sequences recorded during 2 weeks on sample No. 1 which has been kept at low temperatures. Similar histograms were achieved with a lower motion speed of the bridge arms of $17 \mathrm{pm} / \mathrm{s}$.

Several peaks appear for both motion directions at similar positions of $0.8 G_{0}, 1.4 G_{0}$, and $\approx 2.5 G_{0}$. The first peak is split into two substructures at $\approx 0.7 G_{0}$ and $0.9 G_{0}$, a feature which has not been observed for other materials. This splitting has been observed in several independent measurements carried out with four different samples. Although the splitting is observed in all our measurements, the absolute values of the peak positions and the relative peak heights vary slightly from experiment to experiment [see Figs. 2(b) and 2(c)]. A possible explanation of the splitting would be two different configurations of the last contact. This will be discussed in detail below.

The second peak appears already at $1.4 G_{0}$ for both closing and opening traces. This spacing of neighboring peaks is unusually small for metals. The third peak appears to be shifted to slightly lower values $\left(2.2 G_{0}\right)$ in the opening traces as compared to the closing traces $\left(2.5 G_{0}\right)$. In similar experiments by Yanson et al. ${ }^{16}$ on "classical" breakjunctions made of notched Zn wires, no splitting of the first peak is found, but a shoulder that is compatible with our findings. The peaks at higher conductance values are in reasonable agreement with our findings as well. It is well known that details of the histograms do depend on the experimental conditions, as one can easily see by comparing different results of, e.g., the most extensively studied metal gold. In the experiment with $\mathrm{Zn}$ described in Ref. 16 higher voltages have been applied, a slightly higher temperature and faster opening speeds have been used, and only opening traces were recorded. In our experiment, the main difference between opening and closing consists in the peak heights; the low-conductance peaks are less well pronounced when closing. 


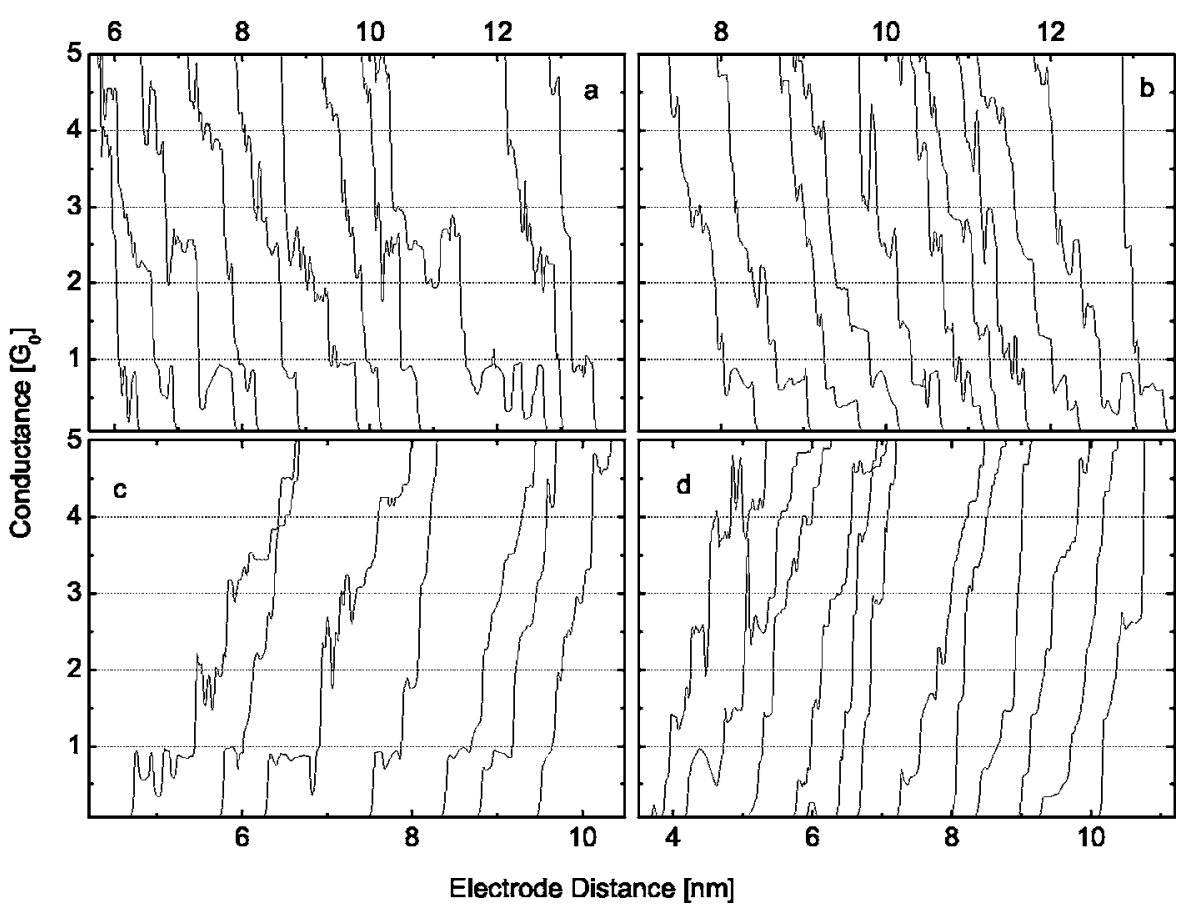

FIG. 3. Opening [panels (a) and (b)] and closing [(c) and (d)] traces of sample No. 1 recorded at 1.4 K. Panels (a) and (c) [(b) and (d)] show examples without (with) the plateau at $1.4 G_{0}$.

\section{B. Individual opening and closing traces}

When analyzing the individual opening and closing traces further, we observe the following correlation: When opening the contact, we either observe jumps from a conductance of around $2.2 G_{0}$ via $0.9 G_{0}$ to almost $0 G_{0}$ or a plateau series $2.5 G_{0}, 1.4 G_{0}, 0.7 G_{0}$ [see Figs. 3(a) and 3(b)]. Only very rarely plateaus at $2 G_{0}$ and $1.4 G_{0}$ exist in the same trace; jumps from $1.4 G_{0}$ to $0.9 G_{0}$ do not occur at all. We also observe jumps directly from values around $2.2 G_{0}$ or slightly above the tunnel regime, but we never observe conductance changes from $1.4 G_{0}$ directly to zero. The plateaus at $2.2 G_{0}$ are usually rather short, while those at $1.4 G_{0}, 0.9 G_{0}$, or $0.7 G_{0}$ are well pronounced. In particular on the $0.9 G_{0}$ plateau we often observe oscillations to much smaller values $\leq 0.4 G_{0}$. About $38 \%$ of all opening curves show the series $2.5 G_{0}, 0.9 G_{0}$ and about the same ratio belongs to the group $1.4 G_{0}, 0.7 G_{0}$. In the closing traces [see Figs. 3(c) and 3(d)] we also often observe the series $0,0.9 G_{0}, 2.5 G_{0}$ or the series $0 G_{0}, 0.7 G_{0}, 1.4 G_{0}, 2.5 G_{0}$, but jumps directly to the $1.4 G_{0}$ or $2.5 G_{0}$ - plateaus are possible. We attribute the latter observation to the formation of contacts larger than a single-atom contact since the apex atoms from the previous opening sequence might have relaxed back to positions deeper in the tip. Also in the closing curves, an oscillating behavior is more pronounced for the $0.9 G_{0}$ plateau than for the $0.7 G_{0}$ plateau. Among all recorded closing traces $48 \%$ show the $0.9-2.5 G_{0}$ plateau series and $28 \%$ the $0.7-1.4 G_{0}$ series. We note that the difference between the opening and the closing histogram is rather small compared to other metals (see, e.g., Ref. 17 for $\mathrm{Au})$.

In summary, there seem to occur preferably two different opening or closing paths, one of which includes geometries giving rise to conductances of 0.9 and $2.2 G_{0}$, while the other one gives conductances of $0.7,1.4$, and $2.5 G_{0}$, respectively.

\section{Histograms of step heights}

The interpretation is further supported by the analysis of the step heights. In Fig. 4 we show a histogram of the stepheights for $\approx 600$ opening (closing) curves. The histogram has been calculated as follows: For each data point (electrode distance $d$, conductance $G$ ) the difference of the conductance to the next data point $\Delta G\left(d_{1}, d_{2}\right)$ (measured in constant time steps of $\Delta t=8 \mathrm{~s}$ ) is determined and a histogram with a bin size of $\Delta G\left(d_{1}, d_{2}\right)=0.1$ is calculated. Since the plateaus in the individual opening (closing) traces are not always clearly marked, small values of $\Delta G\left(d_{1}, d_{2}\right)$ appear more often than larger ones. The overall slope is determined by the opening (closing) speed of the experiment which determines the average change of conductance. If there were no preferred step heights, an exponential decay would be expected. Preferred step heights appear as deviations from this decay. The histogram in Fig. 4 reveals a maximum between 0.7 to $1.0 G_{0}$, but no structure at smaller values. This means

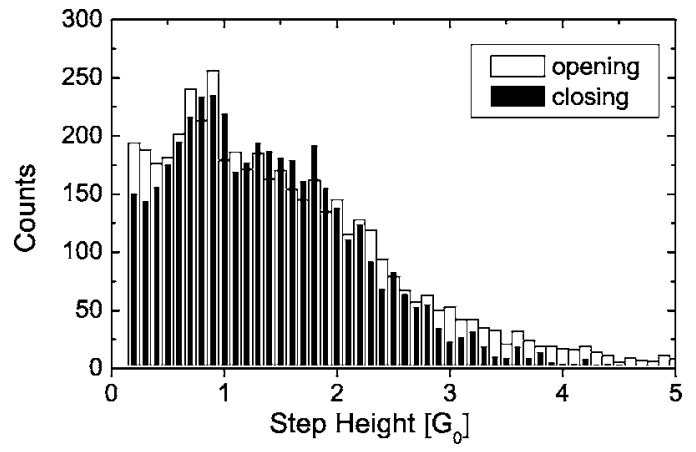

FIG. 4. Histogram of step heights deduced from 600 individual opening and closing curves recorded on sample No. 1, that have been used to calculate the conductance histogram in Fig. 2(a). 
that the rearrangements of the atomic-size contacts preferably change the conductance of the structure by 0.7 to $1 G_{0}$, while smaller jumps are not preferred, in particular jumps between the neighboring peaks in the conductance histogram, e.g., from 1.4 to $0.9 G_{0}$ or 0.9 to $0.7 G_{0}$ or vice versa are not likely to occur. This analysis demonstrates that the conductance histogram is a superposition of two or more histograms with different sets of preferred values.

\section{EXPERIMENTS ON WHISKER MCBS}

\section{A. Single-atom contacts}

Conductance histograms of multivalent metals in general do not show well pronounced multiple peaks. ${ }^{16,18,19}$ An exception of this general observation was so far $\mathrm{Al}$, as mentioned already in the Introduction. The histogram of $\mathrm{Zn}$ shows similarities to that of Al: Several peaks occur with an average spacing between the peaks of about $0.7 G_{0}$. In order to verify whether this similarity would be mirrored in the quantum transport properties, i.e., the conduction channels, measurements on contacts fabricated from whisker breakjunctions have been carried out. The superconducting properties of the lithographic breakjunctions allowed to determine the transport channels of contacts with up to two channels but failed for larger contacts since the superconducting density of states deviates markedly from the BCS shape. ${ }^{20}$ The sample preparation of the whisker MCBs and the results of these investigations together with a detailed theoretical analysis have been reported before. ${ }^{14,20}$ However, the limited lifetime of those contacts hindered the recording of a histogram.

We briefly summarize the main results of Refs. 14 and 20: Although $\mathrm{Zn}$ is a divalent metal, contacts with a dominating single channel, accompanied by a second or even a third one, but with much smaller transmission, similar to the observations for $\mathrm{Au},{ }^{1}$ are observed most often. By a detailed theoretical analysis involving tight-binding calculations of the channels, these observations could be traced back to the symmetry of the valence orbitals $(s$ and $p$ ) of the central atom. These results illustrate that the number of channels in a single-atom contact is not simply determined by the chemical valence, but by the number of valence orbitals together with their symmetry. In particular it could be shown that single atom contacts and dimer contacts grown in the [110] direction would give rise to a smaller overall conductance than the ones grown in the [001] direction. The latter direction was considered in the calculation because it is known to be the preferred growing direction of $\mathrm{Zn}$ whiskers. As explained above, in the lithographical breakjunctions the [001] direction is preferably oriented perpendicularly to the film plane. But the observed nanowires formed between the electrodes might be grown along this direction as well as along the directions in the crystallographic $a-b$ plane, e.g., the [110] direction.

The $I V \mathrm{~s}$ with a dominating single channel are routinely observed at the end of a last plateau after an abrupt change of the $I V$, often including an increase of the conductance, and thus a rearrangement of the atomic-size contact. Once such a contact has been established, it remains stable for an elonga-

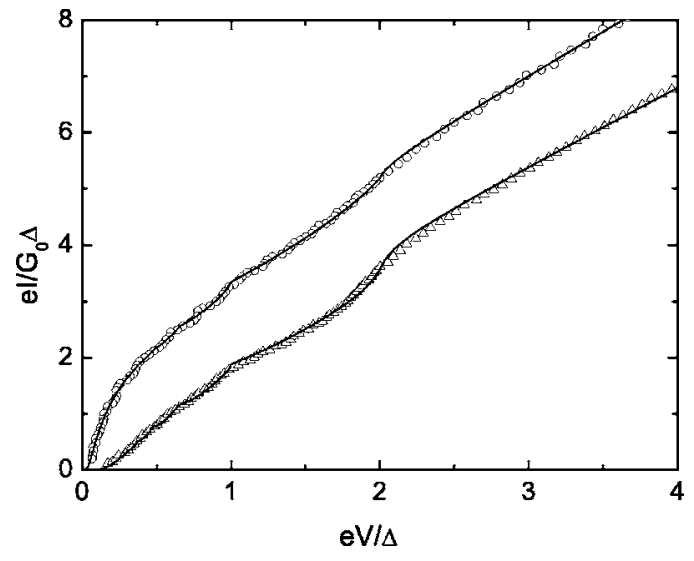

FIG. 5. Current-voltage characteristics of two atomic-size contacts of $\mathrm{Zn}$ arranged with a whisker MCB at $T=0.27 \mathrm{~K}$ (symbols) and best fits according to the theory of MAR (Refs. 21 and 22) (lines) with a superconducting gap value $\Delta=165 \mu \mathrm{eV}$. The transmission coefficients are for the lower curve (triangles): $\tau_{1}=0.82, \tau_{2}=0.25, \tau_{3}=0.19, \tau_{4}=0.09$; and for the upper curve (circles) $\tau_{1}=0.96, \tau_{2}=0.47, \tau_{3}=0.08$. The lower (upper) curve has been measured at position $0.35 \mathrm{~nm}(1.37 \mathrm{~nm})$ of the upper panel of Fig. 6.

tion of the bridge of 2-3 $\AA$. This length compares with the equilibrium nearest-neighbor distance of $2.6 \AA$ of $\mathrm{Zn}$ atoms in the bulk. For Al samples, contacts with a dominating channel with the contribution of one or two smaller channels could be observed as well. ${ }^{4,9}$ However, for Al these contacts evolved continuously from a situation with three channels, occurred only very rarely, ${ }^{17}$ and were immediately destroyed when further pulling the electrodes apart.

\section{B. Larger contacts}

Here we combine the lithographic and the whisker MCB techniques in order to establish a correlation between the atomic configuration and the transport properties. This procedure is justified by the following facts. First, the comparison of individual opening traces shows the same preferred plateau sequences for both types of samples, and second, the transmission coefficients inferred from the lithographical breakjunctions with up to two channels (not shown) are in accordance with those found for the whisker breakjunctions. We have now extended our investigation to larger contacts and the analysis of opening traces.

In Fig. 5 we show two examples of $I V \mathrm{~s}$ measured on a whisker breakjunction at $T=0.27 \mathrm{~K}$ and their fits according to the theory of MAR (Refs. 21 and 22) with three and four channels, respectively. The details of the fitting procedure are described in Ref. 4. The $I V \mathrm{~s}$ are examples recorded on the opening trace shown in Fig. 6 at the positions indicated in the caption. Although the total conductance of the two contacts is similar, $1.37 G_{0}$ and $1.51 G_{0}$, respectively, the $I V \mathrm{~s}$ differ markedly due to the different distribution of transmission coefficients. This example visualizes the possibility to deduce the set of transmission coefficients from the IVs. In the following we will use this knowledge for correlating the structure to the transport behavior. 


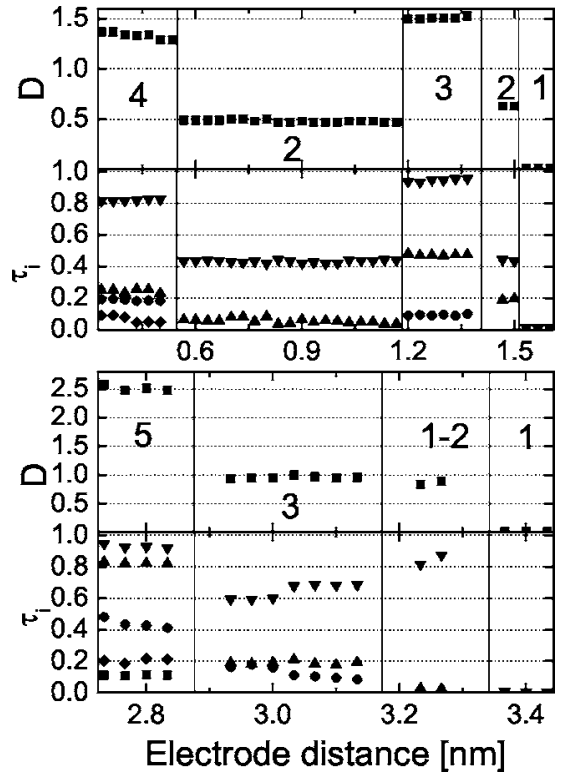

FIG. 6. Upper panel: Opening traces with decomposition into individual channels for a whisker breakjunction showing plateaus at $1.4 G_{0}, 0.5 G_{0}, 1.5 G_{0}$, and $0.6 G_{0}$. Transport direction is presumably close to [001]. Lower panel: Opening traces with decomposition into individual channels for a whisker breakjunction showing plateaus at 2.5 and $1 G_{0}$. Transport direction presumably close to [110]. The origins of the electrode distance axes are at arbitrary values.

Figure 6 shows examples of opening traces recorded on two different whisker MCBs. In the upper panels the total transmission deduced from the conductance is shown, while in the lower panels the decomposition into individual channels is plotted. The example in Fig. 6 (upper panel) shows an opening trace with the typical plateaus at $1.4 G_{0}$ (with four channels) and $0.5 G_{0}$ (with two channels), a reclosure of the contact to $1.5 G_{0}$ (with three channels), and a last plateau at $0.6 G_{0}$ (again with two channels). Although the overall conductance of the starting configuration and the part with three channels is very similar, the channel analysis clearly shows that another atomic configuration is adopted.

Figure 6 (lower panel) depicts an example for the other preferred opening sequence with the plateaus at $2.5 G_{0}$ (five channels) and $1 G_{0}$ (three channels), with an abrupt rearrangement of channels and reduction from three to one (or two) channels within the last plateau. The opening curve displayed in Fig. 6 (lower panel) starts with a conductance of $2.5 G_{0}$ distributed among five channels. The conductance drops then to about $1 G_{0}$ accommodated by three channels. The change of the channel configuration to one dominating channel is hardly seen in the total conductance which remains close to $1 G_{0}$ upon further stretching. However, the $I V$ changes markedly, signalling a distinct atomic rearrangement of the narrowest region. Around the electrode distances $2.9 \mathrm{~nm}$ and $3.15 \mathrm{~nm}$ no stable $I V \mathrm{~s}$ could be recorded because of frequent rearrangements of the contact. For an example of those rearrangements see Ref. 20.

\section{DISCUSSION}

In order to provide further insight into the origin of the sequence of plateaus described above, we have extended the
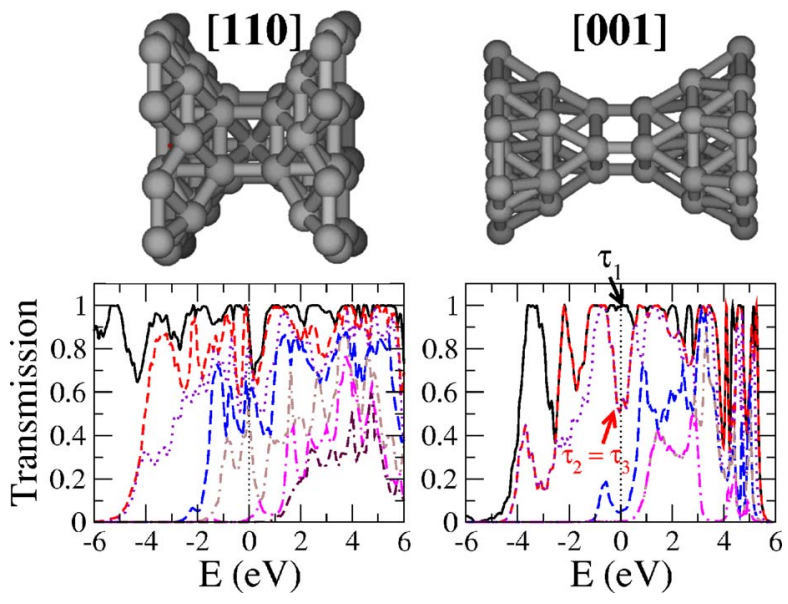

FIG. 7. (Color online) Left-hand panels: The upper one displays a contact grown along the [110] direction with a single central atom. The lower one shows the channel transmissions $\left\{\tau_{\mathrm{i}}\right\}$ as a function of the energy for this contact. The Fermi energy is set to zero and is indicated with a vertical line. The total conductance is $3.6 G_{0}$ and it is dominated by five conduction channels. Right-hand panels: The same as in the left-hand panels for a contact grown in the [001] direction with a minimum cross section of three atoms. The total conductance is $2.1 G_{0}$ with the contribution of four channels. Notice that the second and third channels are degenerate at the Fermi energy.

conductance calculations presented in Ref. 14 to contacts of a larger cross section. These calculations are based on a tight-binding model, which have been described in detail in Refs. 14 and 23. The problem that one encounters in the analysis of contacts larger than the single-atom contact is that the number of possible configurations increases rapidly. We therefore restrict ourselves to only two growing directions and perfectly ordered geometries. For a contact grown in the [110] direction the dimer configuration is the only possibility to have a single-channel contact because already for the single-atom contact shown in Fig. 7 (upper left-hand panel) next-nearest-neighbor contributions of four atoms give rise to five transport channels with non-negligible transmission and a total conductance of $3.6 G_{0}$ (see Fig. 7 lower left-hand panel). The transmissions of those channels vary between 0.5 and 1 . The next larger highly symmetrical contact (not shown here) has four atoms in the smallest cross section with eight channels. Due to the rather large opening angle the number of relevant atoms and channels increases very fast. A change of the separation of the electrodes (for the perfectly ordered geometries used for the calculations) of $0.2 \mathrm{~nm}$ changes the conductance by as much as $2 G_{0}$. Thus, the prediction for this growing direction would be conductance traces with both a rapidly increasing number of channels and rapidly increasing total conductance. In contrast, for the [001] direction, starting again with the dimer configuration with a single channel and transmission $\tau=0.94$, the last but one contact has again one atom in the center of the constriction with one channel and transmission 0.85 , as described in Ref. 14. Upon increasing the disorder the contributions of a second and third channel increase. The smallest highly symmetrical contact with more than one atom in the 
smallest cross section is shown in Fig. 7 (upper right-hand panel) and consists of three atoms with four to five channels and total conductance of $2.1 G_{0}$. From the dimer configuration to this configuration a distance change of more than $0.5 \mathrm{~nm}$ changes the conductance by approximately $1 G_{0}$. Since the absolute values of the transmission coefficients depend crucially on the exact atomic configuration, it is again hard to draw conclusions from the analysis of individual opening traces. Nevertheless, when comparing the results of eight opening traces recorded on two whisker MCBs we find the following trends: The $1.4 G_{0}-0.7 G_{0}$ series gives rise to larger average transmission values than the $2.5 G_{0}-0.9 G_{0}$ series. This sounds contradictory at first sight, but reflects the fact that the larger average conductances in the $2.5 G_{0}-0.9 G_{0}$ series are achieved by more conduction channels. As can be seen in the lower panel of Fig. 6, most of the plateau close to $0.9 G_{0}$ is carried by three channels. In particular, contacts with a dominating single channel are more often observed in the $1.4 G_{0}-0.7 G_{0}$ series, in agreement with the calculations for the [001] direction. ${ }^{14}$ From these considerations we assume the plateau series $2.5 G_{0}-0.9 G_{0}$ to correspond to the [110] direction while for the $1.4 G_{0}-0.7 G_{0}$ the [001] direction seems to be applicable. The exact values of the transmission coefficients, however, differ from the calculated one, presumably because of disorder in the experimental contacts.

\section{SUMMARY}

In conclusion, a single atom or dimer contact of $\mathrm{Zn}$ is likely to have a conductance of $\approx 0.7$ to $0.9 G_{0}$, depending on its geometry. The histograms for both closing and opening the contacts indicate the importance of different contact geometries. By analyzing individual opening and closing traces we identify two preferred plateau sequences. The analysis of the current-voltage characteristics in the superconducting state suggests different preferred transmissions for those typical plateau sequences. By comparing our experimental findings to tight-binding calculations for idealized atomic configurations we propose a correlation between the structure and the transport properties of individual contacts.

\section{ACKNOWLEDGMENTS}

This work was supported by the Deutsche Forschungsgemeinschaft through Grants No. SFB 195, No. SFB 513, and the Alfried Krupp von Bohlen und Halbach-Stiftung. Two of the authors (M.H. and J.C.C.) were supported financially by the Landesstiftung Baden-Württemberg within the "Kompetenznetz Funktionelle Nanostrukturen," the Helmholtz Gemeinschaft (Contract No. VH-NG-029), and the DFG within the CFN.
${ }^{1}$ N. Agraï, A. Levy Yeyati, and J. M. van Ruitenbeek, Phys. Rep. 377, 81 (2003).

${ }^{2}$ R. Landauer, Philos. Mag. 21, 863 (1970).

${ }^{3}$ A. I. Yanson and J. M. van Ruitenbeek, Phys. Rev. Lett. 79, 2157 (1997).

${ }^{4}$ E. Scheer, P. Joyez, D. Esteve, C. Urbina, and M. H. Devoret, Phys. Rev. Lett. 78, 3535 (1997).

${ }^{5}$ H. E. van den Brom and J. M. van Ruitenbeek, Phys. Rev. Lett. 82, 1526 (1999).

${ }^{6}$ R. Cron, M. F. Goffman, D. Esteve, and C. Urbina, Phys. Rev. Lett. 86, 4104 (2001).

${ }^{7}$ B. Ludoph, M. H. Devoret, D. Esteve, C. Urbina, and J. M. van Ruitenbeek, Phys. Rev. Lett. 82, 1530 (1999).

${ }^{8}$ B. Ludoph and J. M. van Ruitenbeek, Phys. Rev. B 59, 12290 (1999).

${ }^{9}$ M. F. Goffman, R. Cron, A. Levy Yeyati, P. Joyez, M. H. Devoret, D. Esteve, and C. Urbina, Phys. Rev. Lett. 85, 170 (2000).

${ }^{10}$ E. Scheer, N. Agraït, J. C. Cuevas, A. Levy Yeyati, B. Ludoph, A. Martín-Rodero, G. Rubio Bollinger, J. M. van Ruitenbeek, and C. Urbina, Nature (London) 394, 154 (1998).

${ }^{11}$ H. Ohnishi, Y. Kondo, and K. Takayanagi, Nature (London) 395, 780 (1998).

${ }^{12}$ V. Rodrigues, T. Fuhrer, and D. Ugarte, Phys. Rev. Lett. 85, 4124
(2000).

${ }^{13}$ D. A. Papaconstantopoulos, Handbook of the Band Structure of Elemental Solids (Plenum Press, New York, 1986).

${ }^{14}$ M. Häfner, P. Konrad, F. Pauly, J. C. Cuevas, and E. Scheer, Phys. Rev. B 70, 241404(R) (2004).

${ }^{15}$ J. M. van Ruitenbeek, A. Alvarez, I. Piñeyro, C. Grahmann, P. Joyez, M. H. Devoret, D. Esteve, and C. Urbina, Rev. Sci. Instrum. 67, 108 (1996).

${ }^{16}$ A. I. Yanson, Ph.D. thesis, University of Leiden, The Netherlands, 2000.

${ }^{17}$ T. Böhler, J. Grebing, A. Mayer-Gindner, H. v. Löhneysen, and E. Scheer, Nanotechnology 15, 465 (2004).

${ }^{18}$ B. Ludoph, N. van der Post, E. N. Bratus, E. V. Bezuglyi, V. S. Shumeiko, G. Wendin, and J. M. van Ruitenbeek, Phys. Rev. B 61, 8561 (2000).

${ }^{19}$ J. L. Costa-Krämer, Phys. Rev. B 55, R4875 (1997).

${ }^{20}$ P. Konrad, P. Brenner, C. Bacca, H. v. Löhneysen, and E. Scheer, Appl. Phys. Lett. 86, 213115 (2005).

${ }^{21}$ D. Averin and A. Bardas, Phys. Rev. Lett. 75, 1831 (1995).

${ }^{22}$ J. C. Cuevas, A. Martín-Rodero, and A. L. Yeyati, Phys. Rev. B 54, 7366 (1996).

${ }^{23}$ J. C. Cuevas, A. L. Yeyati, and A. Martín-Rodero, Phys. Rev. Lett. 80, 1066 (1998). 